\title{
Zur Rolle von Gesten in einer interdisziplinären Unterrichtssequenz Musik
}

\section{Sabine Chatelain}

Aufgrund der Spezifik des Faches spielen im Musikunterricht körperliche Dimensionen eine wichtige Rolle. Sie betreffen sowohl die Musizierpraxis als auch die Werkrezeption sowie den Vermittlungsprozess. Über das Konzept der didaktischen Transposition soll die Rolle von performativen, rezeptiven und didaktischen Gesten als Ausdruck von Körperlichkeit im Musikunterricht untersucht werden. Dazu wurden in einer Fallstudie drei Unterrichtsstunden in einer 9. Klasse (11H) zum Thema "Free Jazz und Action Painting" analysiert deren Besonderheit in einem interdisziplinären Ansatz besteht. Dabei wurde eine klinisch beschreibende Methode angewendet. Die Ergebnisse deuten auf die Notwendigkeit hin, körperliche Aspekte in den verschiedenen Etappen der didaktischen Transposition einschliesslich der vorbereitenden Werkanalyse bewusster herauszuarbeiten.

\section{Einleitung}

Musik ist ein Unterrichtsfach, in dem Aspekte der Körperlichkeit eine grosse Rolle spielen: in der Musikpraxis, beim Musikhören sowie in der Vermittlungssituation. Als Forschungsgegenstand der Musikdidaktik wird Körperlichkeit ins Zentrum einer leibbezogenen anthropologischen Didaktik gerückt (Oberhaus, 2006; Khittl, 2007). Forschungen im Bereich der französischsprachigen Musikdidaktik interessieren sich vor allem für die Rolle des Körpers im Bereich der Musikerausbildung (Mili, Rickenmann, Merchan \& Chopin, 2013; Vitale, 2007). Spezifische Untersuchungen zum Problem gestischer Aspekte in der konkreten Unterrrichtsplanung und - durchführung stehen aber noch aus. Dazu will dieser Artikel einen Beitrag leisten.

Wir beziehen uns auf Vorgehensweisen der vergleichenden Didaktik (Schneuwly, 2013), um uns speziell mit der Problematik der didaktischen Transposition vom Kunstwerk zum Unterrichtsgegenstand zu befassen. Der Fokus liegt hier auf dem Potenzial der Körperlichkeit, welche mehrere Funktionen haben kann. Einerseits wird Musik durch den Körper des Musikers interpre- 
tiert oder geschaffen, hat also eine performative Funktion. Andererseits spielt die Körperlichkeit eine Rolle beim Musikhören, was als rezeptive Funktion bezeichnet werden kann. Zudem hat sie eine didaktische Funktion bei der Übermittlung einer Musikpraxis (Rüdiger, 2007).

Im Folgenden soll erörtert werden, wie sich diese unterschiedlichen Funktionen in der Unterrichtspraxis zeigen. Wir gehen von der Hypothese aus, dass ein besseres Verständnis der körperlichen Aspekte in der didaktischen Transposition neue Einsichten für deren Anwendung in der musikpädagogischen Praxis ermöglichen kann.

Im ersten Teil des Artikels werden verschiedene Aspekte der Körperlichkeit im Zusammenhang mit Musikdidaktik diskutiert, die daran anschliessend auf die Konzepte der didaktischen Transposition (Chevallard, 1985) und Praxeologie (Chevallard, 1992) bezogen werden.

Eine qualitative Analyse des konkreten Unterrichtsgegenstandes - drei Unterrichtsstunden zum Thema "Free Jazz und Action Painting» - wird im zweiten Teil vorgestellt. Die abschliessende Diskussion der Ergebnisse wird mit einem Vorschlag für eine körperlich orientierte Lehr-und Lernperspektive im Musikunterricht verbunden.

\section{Didaktische Transposition von Aspekten der Körperlichkeit im Fach Musik}

\section{Didaktische Transposition}

Über die vergleichende Didaktik wurde das Konzept der didaktischen Transposition in die französischsprachige Musikdidaktikforschung eingeführt, und zum Beispiel in Arbeiten zur kulturellen Rezeption von Musikwerken (Mili \& Rickenmann, 2005) sowie zur Didaktik des Instrumentalspiels (Bourg, 2008) angewendet. Ursprünglich wurde die didaktische Transposition in der Mathematikdidaktik untersucht (Chevallard, 1985). Über dieses Konzept können Unterrrichtshandlungen im Hinblick auf die Wissensvermittlung als sinnstiftender Prozess beschrieben werden. Dabei wird zwischen der geplanten und der realisierten Transposition unterschieden. Der Kontrast zwischen einer ersten, externen Transpositionsphase, d.h. der Vorbereitung und Planung der Unterrichtssequenz, und nachfolgenden internen Transposition gibt Aufschluss über die Bedeutungsangebote, die der Lehrer mit den Schülern tatsächlich umgesetzt hat. Diese Lernangebote können mehr oder weniger körperliche Aspekte beinhalten, die im Folgenden gesondert betrachtet werden sollen. Besonders in künstlerischen Fächern oder im Sportunterricht spielen diese Aspekte eine wesentliche Rolle. Deshalb sollten theoretisches Wissen und die dazugehörige Praxis zusammenhängend betrachtet werden (Martinand, 1986).

Weiterführende Untersuchungen zur didaktischen Transposition stellten die Notwendigkeit heraus, das Zusammenspiel von Wissen und Können zu 
erklären bzw. deren inhärente Logik aufzudecken. Dies kann über Praxeologien (Chevallard, 1992) geschehen, die menschliches Handeln als das Zusammenspiel von Können und den auf dieses Können bezogenen technologischen ${ }^{1}$ und theoretischen Diskurs verstehen. Chevallard stellt heraus, dass jedes [pädagogische] Handeln auch von institutionnellen Logiken bestimmt wird. So wird ein Musikstück im Konzertsaal durch andere Praktiken vermittelt als in der Schule. Sobald ein Musikwerk im Unterricht behandelt wird, wird es für den Zweck des Unterrichts transformiert. Das Konzept der Praxelogie ermöglicht es, Schüler-und Lehrerhandlungen im Zusammenhang mit institutionnellen und persönlichen Konzeptionen der Unterrichtspraxis zu interpretieren.

In unserem Beitrag beziehen wir uns gezielt auf körperliche Aspekte in der Musikdidaktik, die zunächst näher eingegrenzt werden müssen.

\section{Körperlichkeit und Unterricht: Anthropologische Musikdidaktik}

Die Rolle und der Platz der Körperlichkeit wurden vornehmlich in deutschsprachigen Arbeiten zu einer anthropologischen Musikdidaktik erörtert. ${ }^{2}$ Grundsätzlich zielt dieser theoretisch-systematische Ansatz darauf, leibliche Aspekte der Musikwerke und deren Vermittlung herauszuarbeiten, um sie in der Praxis umzusetzen. Khittl (2007) betont, dass schon in der Planungsphase Prioritäten gesetzt werden sollten. So könne eine bewusste Orientierung auf diese Aspekte vor allem beim Musikhören den Schülern gemeinsame Erfahrungen ermöglichen, die nicht auf Expertenwissen aufbauen, sondern auf körperlichem Musikerleben. Auf diese Weise bestehe die Möglichkeit, gängige Hörweisen aufzubrechen, um somit Raum für ungewohnte Höreindrücke zu schaffen. Deshalb kann unserer Meinung nach auch Musik, die der Enkulturation der Hörer nicht entspricht, aus einer neuen Perspektive gehört werden, was unter anderem für Musik des 20. Jahrhunderts relevant ist.

\section{Geste - Körperlichkeit - Leiblichkeit:}

Begriffsbestimmungen

Innerhalb der deutschsprachigen musikdidaktischen Forschung wurde der Begriff der Körperlichkeit mit dem der Leiblichkeit in Bezug gesetzt, um der Ganzheitlichkeit und Bewusstheit des körperlichen Engagements gerecht zu werden (Oberhaus, 2006; Khittl, 2007). Leiblichkeit bezieht sich im phänomenologischen Sinne auf die bewusste Verbindung zwischen Körper und Intellekt. In unserem Verständnis beinhaltet der Begriff Körperlichkeit den Bezug zu bewussten Handlungen. Im Rahmen dieses Artikels wird als beschreibbare Dimension der Körperlichkeit der Begriff der Geste verwendet.

Aufgrund der Bedeutung von Instrumental - und Vokalgesten für die Musikproduktion, kann man Musik aus anthropologischer Perspektive als historisch und sozial determinierte «audible gesture» verstehen (Kogler \& Olive, 2013; Trevarthen, 2000). Als Analogon könnte man bildende Kunst als «visible 
gesture» bezeichnen. Wenn wir Musik und Bilder als Resultat von künstlerisch sowie sozial determinierten Gesten definieren können, so sollte in der Rezeption der Kunstwerke dieser Aspekt eine Rolle spielen. Davon ausgehend ergeben sich im pädagogischen Kontext Konsequenzen für das Verhältnis von Körperlichkeit und Didaktik, wobei im Besonderen auf verschiedene Funktionen von Gesten eingegangen werden soll.

Gesten als gerichtete Aktion und Bewegung haben das Ziel, etwas auszudrücken oder beziehen sich auf den Umgang mit Objekten. Sie übermitteln eine Bedeutung und sind in einen kommunikativen Kontext eingebunden. Musikalische Gesten betreffen sowohl motorische Handlungen beim Musikmachen («musikauslösende und musikbegleitende Bewegungen») als auch innere Nachvollzüge beim Musikhören («innerlich vollzogene Bewegungen») (Gruhn, 2014, S. 28).

Im Musikunterricht findet Lernen über verschiedene Umgangsweisen mit Musik statt: Produktion, Reproduktion, Rezeption, Transposition und Reflexion (Venus, 1984). Im Zusammenhang mit der (Re)Produktion und Rezeption von Musik soll im Folgenden zwischen performativen und rezeptiven Gesten unterschieden werden.

Die performativen Gesten sind die musikauslösenden Spiel - oder Vokalgesten. Sie werden durch musikbegleitende Kommunikationsgesten ergänzt, die sowohl dem musikalischen Ausdruck als auch der nonverbalen Verständigung der Schüler untereinander dienen. Der Prozess der Rezeption von Musik löst einen mimetischen Nachvollzug aus, der auf wahrnehmungspsychologischen Prozessen beruht. Dies zeigt sich beim Musikhören unter anderem über Empfindungen wie Spannung und Entspannung (Imberty, 2005, 2013; Cuen, 2007). Dieser rezeptive Aspekt kann über Körpergesten sichtbar gemacht werden.

Wenn im Unterricht Beispiele oder Techniken über das Zeigen vermittelt werden, bezeichnen wir sie als didaktische Gesten.

Somit ergeben sich verschiedene Kategorien: performative, rezeptive, kommunikative und didaktische Gesten. Wie sich diese im Lehr-und Lernprozess konkret zeigen, soll mit Hilfe des Konzeptes der didaktischen Transposition sichtbar gemacht werden. In diesem Zusammenhang wird zuerst die Rolle der körperlichen Aspekte in der Werkanalyse kritisch hinterfragt.

\section{Körperliche Aspekte der Werkanalyse}

Ein für den Unterricht ausgewähltes Musik- oder Bildwerk existiert vorerst ausserhalb des schulischen Kontextes. Es bedarf einer didaktischen Transposition, um es in schulisches Wissen umzuwandeln. Die vorbereitende Analyse durch die Lehrperson wird in diesem Beitrag als erste Etappe der didaktischen Transposition betrachtet. Für die im Rahmen dieser Untersuchung gewählten Beispiele erschien es notwendig ein Analysemodell vorzuschlagen, das bewusst körperlichen Aspekte der Werke mit einschliesst.

Musik des 20. Jahrhunderts, die weder tonale noch metrische Elemente enthält, kann über übliche Analysekategorien wie Motiv, Harmonie, Rhythmus 
usw. weder adäquat erfasst, noch einfach vermittelt werden, was eine methodische Schwierigkeit darstellt (Bässler, Nimczik \& Schatt, 2004; Floros, 2006). Eine Lösung könnte eine auf gestische Elemente ausgerichte Werkanalyse darstellen (Gritten \& King, 2006).

Gruhns (2004) Schichtenmodell ist zwar auf das ganzheitliche Verstehen von Musik gerichtet, erlaubt aber das Herausstellen gestischer Elemente der Musik. Es unterscheidet wahrnehmungspsychologische, strukturelle und kontextuelle Schichten der Werkrezeption, die als Aussen, Innen- und Tiefenschicht bezeichnet werden. Alle drei Schichten wirken im Verstehensprozess wechselseitig aufeinander und werden nur für ein besseres Verständnis der Werkanalyse getrennt behandelt. Dieses Modell soll hier ebenfalls auf visuelle Elemente angewandt werden.

Klänge oder Farben bzw. Formen, die während der Wahrnehmung direkt körperlich empfunden werden können, werden als Aussenschicht bezeichnet. Sie sind über die Wahrnehmungsorgane direkt erfassbar und können über Körpererfahrungen metaphorisch miteinander in Verbindung gebracht werden (Brandstaetter, 2008; Spampinato, 2013). So sind nicht nur Klänge durch mimetischen Nachvollzug direkt körperlich erfahrbar, sondern auch Bildelemente, die als Bewegung oder Rhythmus leiblich nachvollzogen werden können (Brandstaetter, 2013). ${ }^{3}$

Die Innenschicht bezieht sich auf zugrundeliegende Strukturen des Wahrgenommenen, die erst durch eine eingehendere fachspezifische Analyse bewusst gemacht werden können. Diese Schicht betrifft die spezielle Organisation des Bild- oder Tonmaterials.

Eine dritte Schicht trägt dem historischen und sozialen Kontext der Werkentstehung und seiner aktuellen Rezeption Rechnung. Sie kann über Informationen über das Werk erfasst werden. Je nach der Spezifik der ausgewählten Werke kommt den drei Schichten eine unterschiedliche Rolle zu.

\section{Ziel der Untersuchung und methodisches Vorgehen}

Über die Analyse einer Unterrichtssequenz von drei Stunden in einer neunten Klasse (14 bis 15 jährige Schüler) zum Thema „Free Jazz und Action Painting“ wurde versucht, die unterschiedlichen Aspekte der Gesten zu identifizieren und ihre Rolle im Unterrichtsgeschehen zu analysieren. Die behandelten Werke wurden vom Lehrer ausgewählt, um die Schüler an das Genre Free Jazz heranzuführen. Die Videoaufnahmen der Stunden wurden nach einer klinischbeschreibenden Methode (Leutenegger, 2009) protokolliert und anschliessend analysiert. Die Auswahl der für die Untersuchung relevanten Situationen wurde vom Autor und einer Zweitperson unabhängig voneinander getroffen und anschliessend diskutiert. Der Stundenverlauf wurde in Arbeitsphasen unterteilt, 
die die unterschiedlichen Schüler- und Lehreraktivitäten chronologisch und thematisch gliedern. Die Werkanalyse des Lehrers ergänzt das hier untersuchte Material.

Um die Logik der didaktischen Transposition zu interpretieren, wurde auf das Konzept der Praxeologie zurückgegriffen. Dadurch soll sichtbar gemacht werden, welche Konzeptionen des zu vermittelnden Wissens das Lehrerhandeln bestimmen.

In dieser Studie geht es im Besonderen um die körperlichen Aspekte im Transpositionsprozess. Folgende Fragen sollen beantwortet werden:

- Welche Rolle spielen Gesten im Unterricht?

- Welche gestischen Dimensionen wurden schon in der Werkananlyse hervorgehoben, welche nicht?

\section{Ausgewählte Analysen der Unterrichtssequenz}

Die gesamte Unterrichtseinheit besteht aus folgenden Hauptaktivitäten: Experimentieren von Malgesten nach Musik, musikalische Kollektivimprovisationen, vergleichende Werkbetrachtung und abschliessende Kollektivimprovisation. An vier Beispielen sollen die beobachteten körperlichen Dimensionen des Lehrund Lernprozesses dargestellt werden: die Werkanalyse des Lehrers sowie drei Stundenausschnitte. Alle angeführten Zitate wurden vom Autor ins Deutsche übersetzt. ${ }^{4}$

\section{Körperliche Dimensionen der Werkanalyse}

In der vorbereitenden Analyse der Lehrperson wurden vorrangig musikalische Elemente des Free Jazz herausgearbeitet: das Fehlen von Melodie, Rhythmus und harmonischer Struktur. Dadurch wird dieses Werk in Bezug auf Merkmale tonaler Musik analysiert. Auf gestische Elemente wurde nicht gesondert eingegangen.

Betrachtet man das Stück nun über das Schichtenmodell von Gruhn, so fällt beim Hören ein fast durchgehendes Klangnetz auf, das dem Hörer keine tonalen oder metrischen Anhaltspunkte gibt. Klangfetzen und gehetzte Motive bestimmen das musikalische Geschehen (Aussenschicht). Die Struktur beruht auf einer durchgehenden Kollektivimprovisation von zwei Quartetten (Innenschicht).

In einer Kollektivimprovisation können Spielgesten eine wichtige Rolle spielen, wie es der Komponist und Maler François Nicolas betont:

Eine echte Kollektivimprovisation kann nicht mit einer Komposition verglichen werden. Sie kann keine Direktkomposition sein, da keine komplexe Situation spontan erfunden werden kann; noch weniger kann eine solche Situation weiterentwickelt werden. Sie muss deshalb auf ihre spezifischen Merkmale bauen: das direkte Austesten von Instrumentalgesten der 
Musiker anstatt der Nutzung ausgereifter musikalischer Gesten (Nicolas, 2008, Abs.1). 5

Die erwähnten Schichten, die gestische Dimension sowie der soziale Hintergrund des Free Jazz kommen in der Analyse des Lehrers allerdings nicht zum Tragen.

Den Bezug auf das Werk des Malers Jackson Pollock begründete der Lehrer damit, dass sich Free Jazz und Action Painting gegenseitig beeinflusst haben: Coleman verwendete Pollocks Bild «White light» als Plattencover für sein Werk «Free Jazz» und Pollocks Werke inspirierten später die Musiker des Free Jazz (Tiefenschicht). Die neue Maltechnik des Dripping bringt die ungewöhnlichen Bildstrukturen hervor (Aussenschicht und Innenschicht). Als Element der didaktischen Transposition wurden Ähnlichkeiten im Schaffenprozess ausgewählt. Das Dripping wurde über Colemans Zitat, dass seine Musik so etwas wie Pollocks Malerei sei, mit der Musik in Beziehung gebracht. ${ }^{6}$ Als Lernziel wurde schliesslich die Annäherung an atonalen Jazz über interdisziplinäre und kreative Schüleraktivitäten formuliert. Als gemeinsamer Bezug beider Kunstformen zueinander wurde vom Lehrer das Konzept des Chaos gewählt.

Durch den Bezug auf das Konzept des Chaos bleibt die Werkanalyse auf äussere Merkmale beschränkt, ohne auf die Struktur (Innenschicht) zu verweisen. Gestische Elemente wurden nur im Bezug auf Pollocks Maltechnik angesprochen, die als «das Malen selbst» (la peinture-même) bezeichnet werden können (Guisgand, 2004).Wie im Free Jazz sind hier performative Gesten unmittelbarer Bestandteil des endgültigen Werkes, was jedoch nicht weiter herausgearbeitet wurde.

\section{Visuelle Kollektivimprovisation "Action Painting"}

Die Einstiegsstunde zur Unterrichtseinheit ist dem Thema der bildnerischen Kollektivimprovisation gewidmet. Drei unterschiedliche didaktische Entscheidungen sollen hier kommentiert werden.

In einer ersten freien Improvisation ohne Musik sollten die sechs Gruppen von drei bis vier Schülern Formen generieren, ohne mit dem Pinsel das auf dem Boden liegende A3 Blatt zu berühren. Die gemeinsame Analyse aller Schülerproduktionen wurde nach dem Kriterium der Präsenz erkennbarer Formen durchgeführt.

In der folgenden Übung sollten die Schüler an die Technik des Dripping herangeführt werden. Hier benutzte der Lehrer ein Verbot: das Blatt sollte nicht berührt werden. Es wurden keine weiteren Hinweise zur Maltechnik gegeben. Den Schülern stand es frei, die eingespielte Musik- die ersten fünf Minuten von Free Jazz - als Inspirationsquelle zu nutzen: «Ihr könnt euch von der Musik inspirieren lassen oder auch nicht».

Diese drei didaktischen Entscheidungen (offene Maltechnik, Analyse der Schülerbilder in Bezug auf eine figurale Repräsentation, fehlender Musikbezug) führten unserer Meinung nach zu einer zu offenen Unterrichtssituation. Die 
enge Verbindung zwischen Musik- und Malgeste wurde den Schülern nicht bewusst. Die vorgeschlagene Perspektive der Bildanalyse orientierte die Interpretation der Schüler, sich auf die objektähnliche Formen zu konzentrieren. Auf den Prozess des Malens selbst wurde nicht eingegangen.

Die Technik des Dripping bei Pollock äussert sich über Gesten, die sich aus einer starken Konzentration auf die klangliche Aussenschicht der Musik sowie aus einer gezielten Koordination der Gesten ergeben. Die zur Bewältigung der Aufgabe erforderliche Technik, die kontrollierte Malgeste im Zusammenhang mit der gehörten Musik, konnte durch die Art der Aufgabenstellung nicht gelernt werden. Implizit kamen die Schüler zwar mit ungewohnter Musik in Kontakt, erhielten jedoch keine Hinweise, um über das Dripping auf relevante Indizien der musikalischen Aussenschicht wie Einsatz der Stimmen, Dynamik, Tempo oder Spannungsverläufe zu reagieren.

\section{Musikalische Kollektivimprovisation}

Schüler an eine Kollektivimprovisation heranzuführen, die weder tonale noch rhythmische Anhaltspunkte bietet, ist eine didaktische Herausforderung. In der dritten Unterrichtsstunde (L3, Teil III, 15 - 27 Min.) bekamen die Schülergruppen die Aufgabe, einen kurzen musikalischen Dialog von etwa 30-40 Sekunden zu erfinden. Die Wahl der Instrumente stand ihnen frei. Die Darbietung vor der Klasse erfolgte nach kurzer Besprechung ohne Übungszeit. Diese Aufgabe ist eine Fortsetzung der zweiten Stunde, in der eine erste Gruppenimprovisation erarbeitet und gemeinsam ausgewertet wurde. Dabei wurden folgende Elemente der Musizierpraxis vom Lehrer als bedeutsam herausgestellt: ungewöhnliche Spielgesten, Verwendung rhythmischer Elemente (Ostinato), Synchronisation der Spieler, Dynamik der einzelnen Instrumente und Dialog zwischen den Instrumentalgruppen. Das Konzept des Dialogs wird in der neuen Aufgabenstellung erneut aufgegriffen. Interessant scheint uns hier, die von den Schülern verwendete Technik herauszustellen.

Eine Gruppe von vier Schülerinnen kommuniziert während der Darbietung ihrer Produktion über Gesten wie Blickkontakt, Atmung oder Armbewegungen.

Von Seiten des Lehrers wurde das Konzept des Dialogs als abwechselndes Spielen der Gruppenmitglieder definiert (P, L3, 15'05 Min.). Es blieb aber unklar, welche Strategien angewendet werden können, um den Dialog herzustellen. Der Lehrer rät den Schülern allerdings, sich nicht durch Blickkontakt ablenken zu lasssen (P, L3, 22'50 Min.).

Dem gegenüber dienten die Spielgesten in der beobachteten Gruppe nicht nur der Musikproduktion, sondern gleichzeitig der gegenseitigen Verständigung der Schüler. Der visuelle Bezug auf die Spiel- und Kommunikationsgesten hatte einen wesentlichen Anteil an der Realisierung der Aufgabe, was vom Lehrer nicht hervorgehoben wurde. In den anderen Gruppen konnten die Schüler den Dialog nicht verwirklichen. Dies wurde vom Lehrer zwar erkannt: «Sie sehen [sic] nicht, wie sie sich engagieren können» (CP 35), aber nicht weiter ausgewertet. 


\section{Rezeptive Dimensionen der Körperlichkeit in der Werkbetrachtung}

Die rezeptiven Dimensionen der Körperlichkeit kommen vor allen in der Phase der Werkbetrachtung zum Tragen. Zu Beginn der dritten Stunde wird den Schülern der Kontext der Werke Pollocks und Colemans vorgestellt.

Im Lehrervortrag wird Pollocks Technik des Dripping mit den Gesten eines Dirigenten verglichen: «Ihr habt die gleiche Technik benutzt. Er hat sich von der Musik inspirieren lassen [zeigt eine Dirigiergeste][...] Sein Arm folgt dem Rhythmus der Musik»(P, L3, Teil II/1,5’00 Min.). Der Lehrer zeigt unterschiedliche Dirigiergesten.

Die somit hergestellte Verbindung zwischen Körpergeste, Musik und Malerei beruht jedoch nicht auf einer rezeptiven, sondern auf einer performativen Geste. Ein Dirigent bringt durch seinen Körpereinsatz die Musiker dazu, einen bestimmten Klang zu erzeugen. Im Falle von Pollocks Technik handelt es sich jedoch um die rezeptive Dimension. Die in der Musik enthaltenen Klangelemente der Aussenschicht lösen Körpergesten aus, die dann über Farbe visualiert werden. Durch das Lehrerbeispiel kommt es zu einer Vermischung des Verhältnisses zwischen rezeptiven und performativen Dimensionen der Körperlichkeit.

Anschliessend veranschaulichte der Lehrer das Konzept der freien Improvisation durch die Verfremdung von zwei tonalen Melodien (Bruder Jakob, Happy birthday, L3, Teil II/2, 6'30-8'00 Min.). Die vom Lehrer in Musikfetzen zerlegten Melodien sollen von den Schülern wiedererkannt werden. Der Bezug zum Konzept des Chaos, das sich als Lärm («bruit») äussert, wird aufs Neue in die Argumentation eingebracht.

Durch das Beispiel der Verfremdung bekannter Melodien wird unserer Meinung nach der Bezug zu Coleman's «Free Jazz» nicht klar, da die Jazzmusiker nicht über eine Dekonstruktion bestehender Melodien improvisieren. Ihre sich aufeinander beziehenden Spielgesten werden durch das Beispiel nicht veranschaulicht.

Indem erneut das Chaos als formbildendes Prinzip herausgestellt wird, kann der strukturbildende Charakter der Gesten, welche sowohl beim Hören als auch beim Spielen wichtig sind, von den Schülern nicht bewusst umgesetzt werden.

\section{Diskussion}

Die vorgestellten Resultate sollen nun im Hinblick auf die verschiedenen Funktionen der Gesten in der vorgeschlagenen didaktischen Transposition diskutiert werden.

Performative und didaktische Gesten wurden in allen Stunden beobachtet. Rezeptive Aspekte kommen hingegen nur teilweise in der Werkbetrachtung zu Beginn der dritten Stunde zum Tragen. Auf diese Tatsache soll etwas näher eingegangen werden. 
Das Musikhören, sei es während des Musizierens oder beim Anhören einer Einspielung, wurde nicht bewusst mit körperlichem Erleben musikalischer Gesten in Zusammenhang gebracht. Dieser Umstand wirft die Frage nach dem theoretischen Wissen zu körperlichen Anteilen der Musikrezeption auf, welche zunehmend in wissenschaftlicher Literatur diskutiert wird. Da der mimetische Nachvollzug ein bedeutungsgebendes Element der Wahrnehmung darstellt, sollte dieser Aspekt auch didaktisch umgesetzt werden können. Musikhören als Hauptumgangweise mit Musik könnte somit bewusster mit körperlichen Wahrnehmungen gekoppelt werden. In den untersuchten Unterrichtsausschnitten konnte das Wechselspiel zwischen Musikwahrnehmung und gestischer Umsetzung noch nicht überzeugend gezeigt werden.

Deshalb wurde die Rolle des Körperbezugs in der Werkanalyse genauer betrachtet. Der Vergleich zwischen externer und interner didaktischer Transposition, d.h. zwischen Planung und tatsächlich realisierten Lehr-und Lernvorgängen hat gezeigt, dass die Lehrerhandlungen und der dazugehörige Diskurs nur zum Teil auf körperliche Dimensionen Bezug genommen hatten. Der Vergleich zwischen den Elementen der Werkanalyse und des durchgeführten Unterrichts erwies sich als besonders aufschlussreich. In der untersuchten Sequenz konnte unserer Meinung nach der gegenseitige Bezug zwischen Musik und Malerei nicht schlüssig aufgearbeitet werden, da eine grundsätzliche theoretische Überlegung zu fachübergreifenden Konzepten, wie zum Beispiel zu performativen Gesten, in den kreativen Gestaltungsaufgaben fehlte. Die Auswahl des Unterrichtsgegenstands ist jedoch zu begrüssen, da das Thema Free Jazz als in der Sekundarstufe 1 eher selten behandelt wird.

Die praxelogische Perspektive sollte aufzeigen, wie Praxis und die darunterliegende Theorie zusammenwirken. An den Beispielen des Drippings und der freien Improvisation wurde deutlich, dass eine zu weit gefasste Definition von Kreativität des Lehrers hier nicht zu einer Erweiterung des Ausdruckspotentials der Schüler führte. Solange Kreativität als eine Aktivität betrachtet wird, die keinerlei Regeln bedarf und vor allem auf spontanem Handeln beruht, schliesst diese Haltung gezieltes Erlernen von Techniken aus. Das Beispiel der Kollektivimprovisation, die anscheinend ohne das Erlernen gewisser Regeln auskommt, veranschaulicht diese Praxis.

Die didaktische Entscheidung, Free Jazz als eine Dekonstruktion von tonalen Melodien darzustellen, kann praxeologisch als eine Folge gängiger Musikanalysepraxis erklärt werden, die der fachwissenschaftlichen Ausbildung der Lehrers entspricht. Es stellt sich die Frage, wie eine gestische Analyse komplementär zu traditionnellen Praktiken verwendet werden könnte.

Eine solche Untersuchung würde musikwissenschaftliche und aufführungspraktische Analysen zur musikalischen Gestik (Godoy \& Leman, 2010; Gruhn, 2014) durch eine didaktische Erweiterung dieses Forschungsfeldes sinnvoll ergänzen. 


\section{Synthese}

Diese Untersuchung hatte das Ziel, körperliche Aspekte der didaktischen Transposition im Musikunterricht anhand von Gesten zu untersuchen.

In den ausgewählten Ausschnitten der Unterrichtseinheit zu Free Jazz und Action Painting wurde deutlich, dass den körperlichen Aspekten in der Vorbereitung und Durchführung des Unterrichts verschiedene Funktionen zukommen.

Als Synthese der Ergebnisse ergeben sich zwei Schlussfolgerungen. Einerseits erscheint es sinnvoll, eine vorbereitende Werkanalyse bewussst auf körperliche Aspekte zu orientieren, damit sie beim Klassenmusizieren, Musikhören oder bei der Transformation von Musik in andere Ausdrucksformen didaktisch bewusst genutzt werden können. Andererseits könnten die theoretischen Arbeiten zur Rolle von Gesten beim Musiklernen und -verstehen für didaktische Überlegungen gezielter eingesetzt werden.

Um weiteren Aufschluss über die spezifischen Rollen der körperlichen Aspekte im Musikunterricht zu bekommen, könnte die hier aufgezeigte Verbindung zwischen vorbereitender Werkanalyse, Unterrichtsplanung und-durchführung weiterverfolgt werden. Damit könnten weitere Anstösse für didaktische Überlegungen gegeben werden, die der Rolle der Körperlichkeit in den Phasen der Werkanalyse, der Produktion sowie der Rezeption eine grössere Bedeutung schenken.

\section{Notes}

1 Chevallard bezeichnet Technologie als den erklärenden Diskurs, den logos.

2 Wir beziehen uns hauptsächlich auf die Arbeiten von Oberhaus (2006) und Khittl (2007) zum Entwurf einer anthropologischen Musikdidaktik.

3 Ursula Brandstaetter bezieht sich auf Bernhard Waldenfels, der in seinem Aufsatz «Verkörperung im Bild» den medialen, szenischen und pathischen Charakter der Bilderfahrung herausarbeitet. Einen weiteren Ansatz zum intermedialem Verstehen von Musik und bildendender Kunst bietet die Arbeit von Spampinato (2011), der Musik von Debussy mit Malerei des Impressionismus über Metaphern der körperlichen Wahrnehmung vergleicht.

4 Verwendete Abkürzungen: $\mathrm{L}=$ leçon/Stunde; $\mathrm{CP}=$ commentaire du professeur/Lehrerkommentar; $\mathrm{E}=$ élèves/Schüler; $\mathrm{P}=$ professeur/Lehrer

5 «Une improvisation collective qui ne triche pas ne saurait rivaliser avec la composition. Elle ne saurait être une composition instantanée car elle ne saurait inventer instantanément une situation complexe, originalement stratifiée; moins encore, elle ne saurait la développer. Elle doit donc jouer de ses atouts spécifiques: l'exploration instantanée de gestes musiciens (instrumentistes) plutôt que l'exploitation longuement mûrie de gestes musicaux."

6 Da alle hier angeführten Argumente auf französisch formuliert wurden, verzichten wir im Rahmen dieser Analyse weitgehend auf wörtliche Zitate. 


\section{Literatur}

Bässler, H., Nimczik, O. \& Schatt, W. P. (2004). Neue Musik vermitteln. Analysen-Interpretationen-Unterricht. Mainz: Schott.

Bourg, A. (2008). Didactique de la musique: Apports d'une approche comparatiste. Education et didactique, 2(1), 69-88. Zugriff am 5.10.2014 unter http://educationdidactique.revues.org/

Brandstaetter, U. (2008). Grundfragen der Ästhetik. Bild-Musik-Sprache-Körper. Köln, Weimar, Wien: Böhlau.

Brandstaetter, U. (2013). Erkenntnis durch Kunst. Theorie und Praxis der ästhetischen Transformation. Weimar, Wien, Zürich: Böhlau.

Chevallard, Y. (1985). La transposition didactique. Du savoir savant au savoir enseigné. Grenoble: La Pensée Sauvage.

Chevallard, Y. (1992). Concepts fondamentaux de la didactique : perspectives apportées par une approche anthropologique. Recherches en didactiques des mathématiques, 12(1), 73-111.

Cuen, L. (2007). Le geste sonore et le geste cinétique: Une évocation des sensations et des émotions humaines. In M. Imberty \& M. Gratier (Éd.), Temps, Geste et musicalité (pp. 151-167). Paris: L'Harmattan.

Floros, C. (2006). Neue Ohren für neue Musik. Mainz: Schott.

Godoy, R.- I. \& Leman, M. (Ëd.).(2010). Musical gestures. Sound, mouvement and meaning. New York, London: Routledge.

Gritten, A. \& King, E. (Éd.).(2006). Music and gesture. Aldershot: Ashgate.

Gruhn, W. (2004). Wahrnehmen und Verstehen. Wilhelmshafen: Florian Netzel.

Gruhn, W. (2014). Musikalische Gestik. Hildesheim, Zürich, New York: Olms.

Guisgand, P. (2004). Pollock ou les états de corps du peintre. Revue DEMéter, Université de Lille, 1-11. Zugriff am 20.11.2014 unter http://demeter.revue.univ-lille3.fr/corps/ guisgand

Imberty, M. (2005). La musique creuse le temps. L'Harmattan: Paris.

Imberty, M. (2013). Mouvement, geste et figure : la musique ancrée dans le corps. In S. Kogler, S. \& J.-P. Olive (Éd.), Expression et geste musical (pp. 25-36). Paris: L'Harmattan.

Leutenegger, F. (2009). Le temps d'instruire. Approche clinique et expérimentale du didactique ordinaire en mathématiques. Bruxelles: Peter Lang.

Khittl, C. (2007). «Die Musik fängt beim Menschen an»: Anthropologische Musikdidaktik, theoretisch-praktisch. Bern: Peter Lang.

Kogler, S. \& Olive, J.-P. (Éd.), (2013). Expression et geste musical. Paris: L'Harmattan.

Martinand, J.-L. (1986). Connaître et transformer la matière: des objectifs pour l'initiation aux sciences et techniques. Bern: Peter Lang.

Mili, I. \& Rickenmann, R. (2005). La réception des œuvres d'art : une nouvelle perspective didactique. Schweizerische Zeitschrift für Bildungswissenschaften, 27 (3), 431-452.

Mili, I., Rickenmann, R., Merchan Price, C. \& Chopin, M.-P. (2013). Corps sonore/corps scénique/corps écrit: Corporéité dans les didactiques des arts (dans, théâtre, musique, arts visuels). In J.-L. Dorier, F. Leutenegger \& B. Schneuwly (Éd.), Didactique en construction, construction des didactiques (pp. 87-108). Bruxelles: De Boeck.

Nicolas, F. (2008). De l'improvisation collective. Zugriff am 12.06.2013 unter http://www. entretemps.asso.fr/Nicolas/Improvisation/Propositions.html

Oberhaus, L. (2006). Musik als Vollzug von Leiblichkeit: Zur phänomenologischen Analyse von Leiblichkeit in musikpädagogischer Absicht. Essen : Die blaue Eule.

Rüdiger, W. (2007). Der musikalische Körper. Mainz: Schott.

Schneuwly, B. (2013). Didaktik: Aufbau eines disziplinären Feldes - Eine frankofone Perspektive. Beiträge zur Lehrerbildung, 31(1), 18-30.

Spampinato, F. (2011). Corps, synesthésie, homologie. Debussy entre impressionnisme et symbolisme. In M. Barbe (Éd.), Musique et arts plastiques. La traduction d'un art par l'autre (pp. 87-99). Paris: L'Harmattan. 
Trevarthen, C. (2000). Musicality and the Intrinsec Motor Pulse. Musicae Scientiae

Special Issue, 155-211.

Venus, D. (1984). Unterweisung im Musikhören. Wilhelmshafen: Heinrichshofen.

Vitale, A. (2007). Geste et mémoires dans l'apprentissage du chant. In M. Imberty \& M.

Gratier (Éd.), Temps, Geste et musicalité (pp. 101-126). Paris: L'Harmattan.

Schlagworte: Geste, Musikhören, Interdisziplinarität, Kollektivimprovisation,

Didaktische Transposition

\section{À propos du rôle des gestes dans une séquence d'enseignement interdisciplinaire en musique}

\section{Ré sumé}

En éducation musicale, les dimensions corporelles jouent un rôle important en raison de la spécificité de la discipline. Ces dimensions concernent la pratique, la réception et les processus d'enseignement-apprentissage de la musique. À travers la notion de transposition didactique, nous souhaitons identifier le rôle des gestes performatifs, réceptifs et didactiques dans cet enseignement. Dans une classe de $11 \mathrm{H}$, nous avons analysé trois leçons portant sur la thématique interdisciplinaire «Free Jazz et Action Painting» en utilisant une méthode clinique. Les résultats suggèrent de tenir compte des aspects de corporéité dans les différentes phases de la transposition didactique, y compris lors de l'analyse préalable des œuvres.

Mots-clés: Geste, écoute musicale, interdisciplinarité, improvisation collective, transposition didactique

\section{II ruolo dei gesti in una sequenza d'insegnamento interdisciplinare in Educazione Musicale}

\section{Riassunto}

Le dimensioni corporee giocano un ruolo importante in Educazione Musicale proprio per la specificità di questa disciplina. Queste dimensioni comprendono la pratica, la ricezione e il processo di insegnamento-apprendimento della musica. Attraverso il concetto di trasposizione didattica, desideriamo identificare il ruolo dei gesti performanti, ricettivi e didattici nell'insegnamento di questa materia. In una classe di undicenni, abbiamo analizzato con un metodo clinico tre lezioni sulla tematica interdisciplinare "Free Jazz et Action Painting". I risultati invitano a tener conto degli aspetti della corporalità, nelle differenti fasi della trasposizione didattica, inclusa la premessa nell'analisi delle composizioni.

Parole chiave: Gesto, ascolto musicale, interdisciplinarità, improvvisazione collettiva, trasposizione didattica 


\section{The function of gestures within an interdisciplinary teaching sequence in music}

\section{Summary}

Due to specific features of music, bodily dimensions play an important role within music education. These dimensions concern music practicing, music reception, as well as the music teaching and learning process. With respect to the concept of didactic transposition, we examine the role of bodily, receptive and didactical gestures within music education. Our research has been carried out with 14-15 year-old pupils participating at a case study including three interdisiplinary lessons on the theme «Free Jazz and Action painting». Results suggest to use gestures more consciously during all the steps of the didactical transposition process, including during the a priori analysis of the art work.

Keywords: Gesture, listening, interdisciplinarity, group improvisation, didactical transposition 